\title{
Carcinoid heart disease and recurrent hypertensive crises in a patient with primary high-grade ovarian neuroendocrine carcinoma
}

\author{
Adam M Brouillard ${ }^{1 *}$,John M Kasznica ${ }^{2}$ and Jennifer K Lang ${ }^{1}$ \\ ${ }^{1}$ Department of Medicine, Division of Cardiology, Jacobs School of Medicine and Biomedical Sciences, Buffalo, USA \\ ${ }^{2}$ Roswell Park Cancer Institute, Buffalo, USA
}

\begin{abstract}
Background: Carcinoid tumors compose approximately $0.1 \%$ of all ovarian carcinomas and can cause carcinoid syndrome and valve disease without metastases due to systemic venous drainage bypassing liver metabolism. Carcinoid valve disease can result in significant clinical heart failure despite it being typically well tolerated clinically. Hypertensive crisis is a potential presentation of carcinoid syndrome, but it is not well described in the literature.

Case: We present a case of a 41-year-old African American female who developed carcinoid heart disease from a primary high grade left ovarian neuroendocrine carcinoma. The patient was referred to our cardiology clinic for new onset, refractory hypertension to multiple anti-hypertensive agents as well as 30-pound weight gain and symptoms of carcinoid syndrome and congestive heart failure. Echocardiogram revealed moderate tricuspid leaflet and annulus thickening with reduced tricuspid opening and turbulent high-velocity diastolic flow across the tricuspid valve. Serum serotonin was markedly elevated at 2,237 . The patient underwent surgical resection of the left adnexal mass found on pelvic computed tomography scan. Frozen section showed stage 1A, grade 3 neuroendocrine ovarian carcinoma. On follow-up, the patient experienced resolution of her preexisting uncontrolled hypertension, carcinoid syndrome and heart failure symptoms. Her valvular disease has not progressed on serial clinical and echocardiographic follow-up.

Conclusion: Carcinoid syndrome is an extremely rare cause of secondary hypertension, particularly in patients without hepatic metastases. In this case, we present a patient with primary ovarian neuroendocrine carcinoma with presenting symptoms most notable for hypertensive crises, which to the best of our knowledge has not been reported. Carcinoid tumors should be considered in patients with new-onset, poorly controlled hypertension, particularly in females where ovarian tumors' ability to bypass portal circulation can lead to rapid progression of disease.
\end{abstract}

\section{Background}

Carcinoid tumors are neuroendocrine malignancies composed of enterochromaffin cells most commonly derived from the gastrointestinal tract [1]. These tumors are known to cause carcinoid syndrome by a mechanism that is not fully understood, although significant amounts of serotonin are produced leading to symptoms including wheezing from bronchoconstriction, flushing, diarrhea and right-sided heart valve disease [2]. The overall incidence of carcinoid tumors is estimated to be between 1-2 case in 100,000 people [3]. Carcinoid tumors derived from the ovary are exceedingly rare, with estimates of $0.31 \%$ and $0.52 \%$ among all carcinoid tumors [4,5]. Within the ovary, carcinoid tumors compose approximately $0.1 \%$ of ovarian carcinomas [6]. Although hypertension and hypotension have been described as potential symptoms of carcinoid syndrome [6], to our knowledge there are no documented reports of repeated hypertensive crises as the primary presenting symptom in this condition.

\section{Case report}

This is a case of a 41-year-old African American female with a past medical history notable only for intermittent asthma and chronic anemia who was referred to our outpatient cardiology clinic. The patient was referred by her primary physician secondary to new and sudden onset generalized edema and poorly controlled hypertension despite the use of several anti-hypertensive medications. In the clinic, she described a 5-month history of generalized edema involving her face, upper and lower extremities, which resulted in a 30-pound weight gain. Additionally, she complained of diarrhea, flushing, pruritus and congestive heart failure symptoms including dyspnea on exertion, paroxysmal nocturnal dyspnea and orthopnea (New York Heart Association Class IV). She denied the presence of a rash and did not have an increased need for her albuterol inhaler. She also detailed intermittent episodes of severe headaches and blurry vision that prompted multiple visits to the Emergency Department where her systolic blood pressure was found to be greater than $200 \mathrm{~mm} \mathrm{Hg}$. She did not have a preexisting history of hypertension until 6 months prior and in the past 5 months, her primary physician had been unable to control her blood pressure with initiation and up-titration of clonidine, Lisinopril and furosemide. Her medications also included ferrous sulfate $325 \mathrm{mg}$ three times daily and potassium chloride 20

Correspondence to: Adam M Brouillard, Department of Medicine, Division of Cardiology, Jacobs School of Medicine and Biomedical Sciences, Buffalo, N.Y, 14203, USA.

Key words: carcinoid heart disease, hypertensive crisis, neuroendocrine carcinoma, tricuspid valve

Received: November 18, 2016; Accepted: December 09, 2016; Published: December 12, 2016 
mEq daily. Her only known allergy was to shellfish. The patient denied any precipitating events, prodromal viral symptoms, environmental exposures or travel. Further history revealed no tobacco or alcohol use and a family history significant on her paternal side for hypertension, diabetes mellitus and coronary artery disease.

On physical exam, the patient had a blood pressure of 167/104 in the right arm with a $2+$ radial pulse and a rate of 69 . On the left side, the diastolic pressure was 84 and the radial pulse was $1+$. The exam was also significant for diffuse edema, most notable in the face, arms and distal lower extremities. There was no edematous involvement of the tongue or lips. On cardiac exam, there was a grade 2 pansystolic murmur best heard in the left lower sternal border which increased with inspiration. The lung exam revealed no wheezes, rales or rhonchi. The remainder of the physical exam was not significant. An electrocardiogram revealed no significant abnormalities. Initial blood work showed a WBC of 6.1, hemoglobin of 11.8 , hematocrit of $37.1, \mathrm{MCV}$ of 75.5 , platelets of 317,000 , normal coagulation profile, electrolytes, kidney function and liver function. Troponin was less than 0.01 , creatine kinase was 65 , BNP was 165 and myoglobin was 14 . Prior imagine studies included a retroperitoneal ultrasound that showed a $25 \mathrm{~mm}$ hyperechoic area within the mid to upper pole of the left kidney as well as a transthoracic echocardiogram (Figure 1A-D). The echocardiogram was notable for a left ventricular ejection fraction of $70 \%$ without any obvious wall motion abnormalities. There was moderate tricuspid leaflet and annulus thickening with reduced tricuspid opening and turbulent high-velocity diastolic flow across the tricuspid valve consistent with moderate tricuspid regurgitation and mild tricuspid stenosis. Additionally, the patient was found to have mild pulmonary hypertension with a right ventricular systolic pressure of $35 \mathrm{mmHg}$. Further findings included a small posterior pericardial effusion with mild pericardial and epicardial thickening without signs of impending hemodynamic compromise. There was no prior echocardiogram available for comparison.

Due to the patient's new onset and poorly controlled hypertension in the presence of her associated symptoms, a secondary cause of her hypertension was suspected. To evaluate her hypertension, thyroid stimulating hormone (TSH), free T4, serum cortisol, serum serotonin and 24-hour urine collection of protein and catecholamines
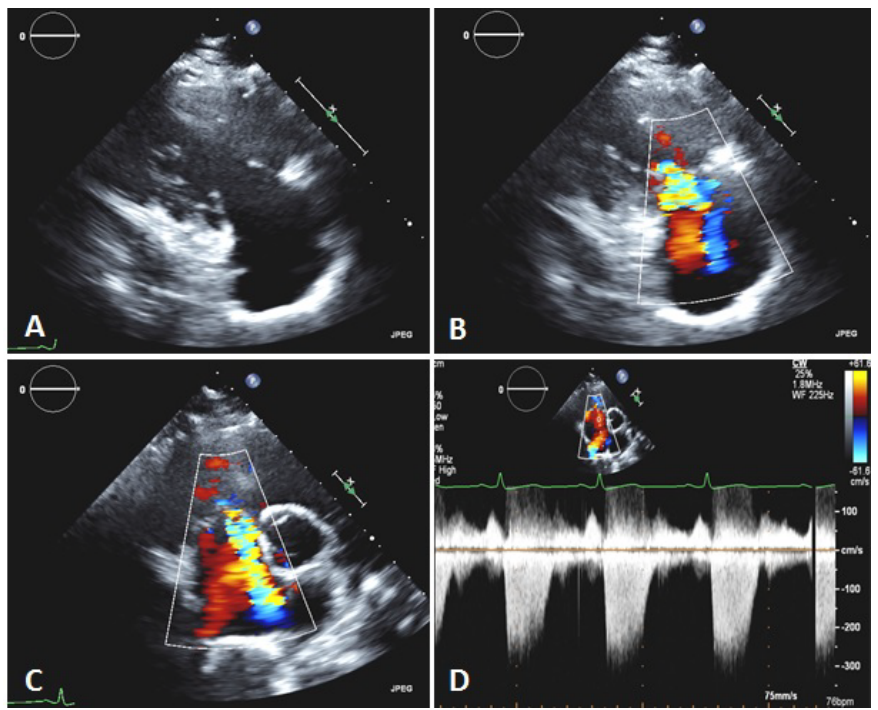

Figure 1. Transthoracic echocardiography showing thickened and retracted tricuspid valve leaflets (A) with associated moderate tricuspid regurgitation, shown by color Doppler (RV inflow, B; parasternal short axis, C) and continuous wave Doppler (D).

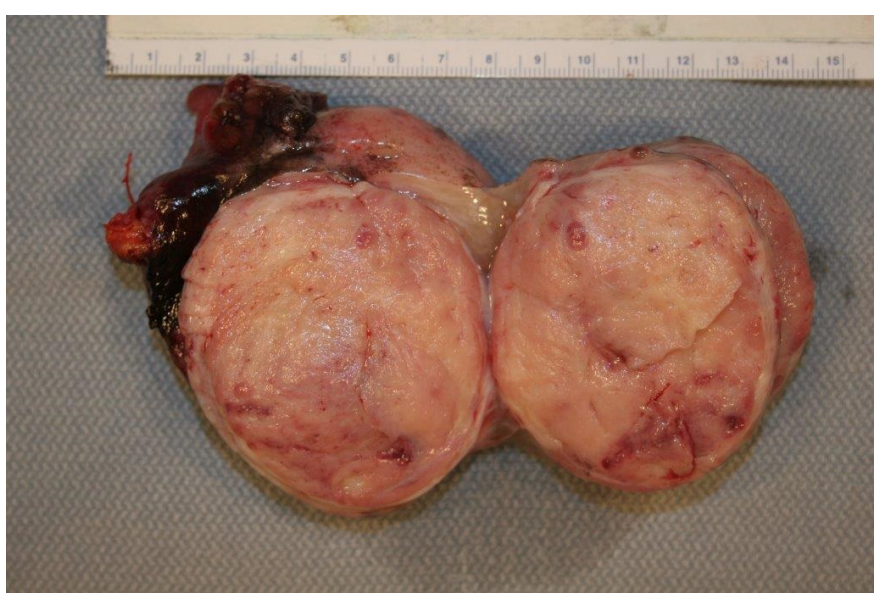

Figure 2. The left ovary $(8.1 \mathrm{~cm})$ was entirely replaced by a predominantly fleshyappearing tumor. The outside ovarian capsule was smooth and there was no gross evidence of penetration of the capsule or outside growth of the tumor on the ovarian surface. Gross photograph.

were ordered. The patient's cortisol was found to be elevated, but most notably, her serotonin level was found to be very elevated at 2,237 (normal upper limit of 420). A renal Doppler ultrasound was performed, which revealed no hemodynamically significant renal artery stenosis. An abdominal and pelvic computed tomography (CT) scan was done, which showed a left adnexal mass with cystic and solid components. The mass measured $8.5 \times 8.2 \times 7.4 \mathrm{~cm}$ on CT and was not present on a CT that the patient had four years prior to the current study. Given the significantly elevated serotonin and newly discovered adnexal mass, the patient was emergently referred to gynecologic oncology for further evaluation.

The patient was evaluated by a consulting gynecologic surgeon who performed an exploratory laparotomy with suspicion of a carcinoid tumor or neuroendocrine carcinoma. The procedure ultimately resulted in a total abdominal hysterectomy, bilateral salpingooopherectomy, bilateral pelvic and para-aortic lymphadenectomy, partial omentectomy and appendectomy. The procedure was complicated by hypertension that reached as high as $270 / 191$, but no sequelae were found following the surgery. The patient was discharged post-operative day 6 to home in stable condition with improved blood pressure control. The left ovary was completely replaced by tumor, which measured $8.1 \mathrm{~cm}$ in maximum dimension (Figure 2). The surface of the ovary did not show penetration of the tumor through the ovarian capsule. On cut section, the tumor was tannish-gray to pink with some hemorrhagic areas and somewhat fleshy in consistency. The remaining specimens removed at the time of surgery (uterus, cervix, contralateral adnexa, staging lymph nodes and appendix) did not show evidence of tumor. On microscopic examination, the tumor grew in sheets and cell groups revealing a trabecular growth pattern. It did not form definite glands or show evidence of keratinization and did not show evidence of melanin pigment (Figure 3). The cells, including their nuclei, had a neuroendocrine appearance, and showed high grade cytological and histological features, which were morphologically consistent with a high grade neuroendocrine carcinoma (Figure 3). AE1/AE3 stain positivity was supportive of the epithelial character of this tumor (i.e., carcinoma). CD56, synaptophysin, and chromogranin were all positive, which confirmed the morphologically suspected neuroendocrine nature of the tumor (Figure 4). The tumor was diagnosed as a stage 1A, grade 3 neuroendocrine ovarian carcinoma. The morphology of the tumor was noted to be different from hypercalcemic type neuroendocrine 


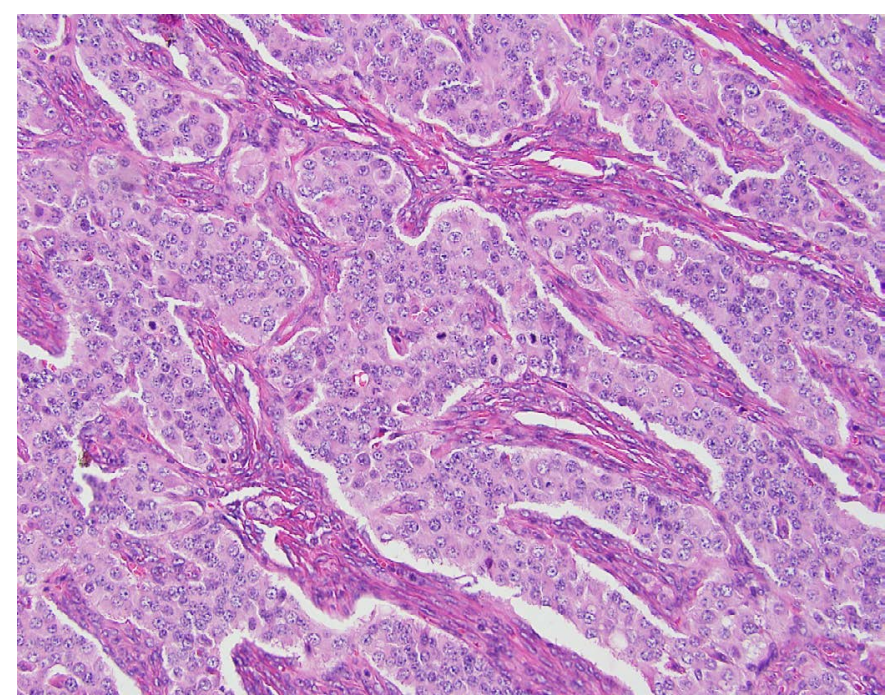

Figure 3. Medium power view of the tumor architecture and cytological characteristics. The tumor shows groups of neoplastic cells, growing in a solid pattern. No glands, tubules or ducts were seen. There was no keratinization, and there was no melanin pigment seen. A trabecular growth pattern of the tumor was noted. The nuclear and cytological features were those of a high grade neuroendocrine carcinoma. The immuno-histochemical panel results supported the diagnosis of high grade neuroendocrine carcinoma. HematoxylinEosin stain, original magnification $200 x$.

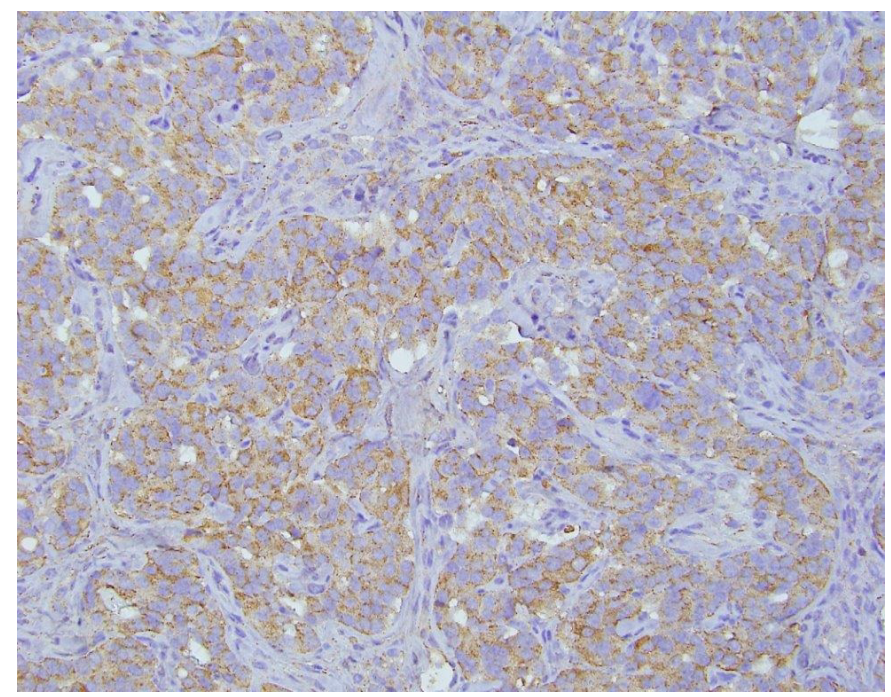

Figure 4. Medium power view of the neoplasm stained by Chromogranin A immunehistochemical stain, showing the brownish positive reaction of the immuno-peroxidase stain in a trabecular growth pattern. Synaptophysin and CD56 were also strongly positive consistent with the neuroendocrine nature of this neoplasm. Chromogranin A stain, original magnification 200x.

carcinoma, as well as typical and atypical carcinoid tumors. It was noted that it is unusual for high grade neuroendocrine carcinomas to secrete serotonin, which typically occurs in low grade carcinoid tumors. The patient completed six courses of adjuvant chemotherapy with etoposide and cisplatin, which was well tolerated.

The patient was seen one year after her initial evaluation in the cardiology clinic. She complained of none of her initial symptoms and reported a 30-pound weight loss after her surgery that returned her to her baseline weight prior to the onset of her symptoms. Repeat echocardiogram showed a left ventricular ejection fraction of 60 $65 \%$ with normal right ventricular function and no wall motion abnormalities. The pulmonary artery systolic pressure was measured at $48 \mathrm{~mm} \mathrm{Hg}$. Her tricuspid valve showed mild tricuspid stenosis and moderate tricuspid regurgitation with diffuse thickening of the anterior leaflet and decreased diastolic leaflet excursion. Her blood pressure was well controlled at 140/80 with amlodipine $5 \mathrm{mg}$ daily, hydrochlorothiazide $25 \mathrm{mg}$ daily and Lisinopril $20 \mathrm{mg}$ daily. On cardiac exam, a II/VI holosystolic murmur was auscultated at the left parasternal border. Her physical exam was otherwise unremarkable. Surveillance imaging had shown no evidence of residual cancer at two years after surgery. Oncologic follow-up was planned for a duration of five years. For her valvular disease, the patient continues to be evaluated with serial echocardiograms at one year intervals. Since there was no progression of her valvular disease and her heart failure symptoms had resolved, no intervention to repair the tricuspid valve was deemed necessary. She continues to follow-up in our cardiology clinic with no worsening of her disease clinical and echocardiographic evaluation.

\section{Discussion}

The first description of the occurrence of a primary ovarian carcinoid tumor was reported in 1939 by Stewart et al. [7] with the first recognition of carcinoid syndrome and heart murmur from a primary ovarian carcinoid tumor occurring in 1958 by Sauer et al. [8]. Among ovarian carcinoid tumors, approximately $33 \%$ of cases lead to clinically significant carcinoid syndrome [6]. In a large analysis of carcinoid tumors, $85 \%$ of ovarian cases were found in Caucasian patients, with only $12.5 \%$ in African American patients like the case described above [4]. Carcinoid tumors are typically diagnosed in patients with ages greater than our reported case (age 41), with a reported average age of 55.3 years.

The vast majority of carcinoid tumors originate in areas outside of the ovary, most notably, the appendix, ileum, rectum and lung [4,5]. Tumors within the gastrointestinal tract typically must metastasize to the liver before symptoms are evident because of the hepatic clearance of tumor metabolites in portal venous circulation. However, Bernheim et al. reported a four patient case series of carcinoid tumors without ovarian or liver involvement that developed valve disease postulated to be due to lymphatic spread through the thoracic duct [2]. Ovarian carcinoid tumors are unusual in their pathophysiology because venous drainage bypasses the portal circulation and the liver via the renal vein on the left and the inferior vena cava on the right. This anatomy allows ovarian tumors to present with early carcinoid symptoms without the need for liver metastases. Chaowalit et al. have previously reported four cases of these rare ovarian tumors where all patients required tricuspid valve replacement and surgical tumor removal [9]. All four patients did not present until severe valve disease was found, and three of the patients were elderly [9], which differs from the presentation of our case. Our patient reported NYHA Class IV heart failure symptoms despite moderate valvular disease, which is unusual as carcinoid heart disease typically presents with NYHA Class I symptoms despite severe valve lesions [1]. Therefore, clinicians may consider carcinoid heart disease when a patient presents with rapidly progressive heart failure without an identifiable cause.

It has been estimated that up to $20 \%$ of patients with carcinoid syndrome present with valvular heart disease at diagnosis [1]. In the evaluation of carcinoid heart disease, transthoracic echocardiogram is considered essential to diagnosis with chest $\mathrm{x}$-ray and electrocardiogram lacking sensitivity in these cases [1]. Brain Natriuretic Peptide (BNP) has been found to be elevated in carcinoid tumor patients with carcinoid heart disease, as seen in our patient, as compared to carcinoid tumor patients without cardiac involvement, 
but this finding lacks sensitivity and specificity [1]. Characteristic echocardiographic findings in carcinoid heart disease include plaquelike, fibrous endocardial thickening that classically involves the right side and causes retraction and fixation of the leaflets [3]. In a series of 74 patients, Pellikka et al. evaluated echocardiographic findings of carcinoid heart disease and characterized tricuspid valve disease more specifically [10]. In patients who had Doppler studies performed, all patients had tricuspid insufficiency, which was more prominent in the septal and anterior leaflets, and a dagger-shaped spectral profile in continuous wave Doppler was described [10]. This description is consistent with our reported patient who developed thickening most prominently on the anterior tricuspid leaflet.

In patients with carcinoid syndrome, typical symptoms include flushing (78\%), diarrhea (61\%) and wheezing (21\%) and valve disease $(20 \%)$ [1,11]. Our patient presented with flushing, diarrhea and tricuspid valve disease. It is plausible that she was experiencing wheezing related to carcinoid syndrome, but this feature was difficult to determine given the patient's underlying asthma. It is noteworthy that within the literature of carcinoid tumors and carcinoid syndrome, there is minimal inclusion of blood pressure symptoms in the presentation of this diagnosis. Our patient's primary complaints and symptomatology was directly related to her difficult to control hypertension and subsequent edema, headaches and blurry vision. It has been noted that carcinoid syndrome stemming from an ovarian carcinoid tumor is an under investigated etiology of right-sided valvular disease in women [9]. Our patient experienced several episodes of hypertensive crisis that resulted in Emergency Department presentations for severe headaches and significantly elevated systolic blood pressure greater than 200 $\mathrm{mm} \mathrm{Hg}$. We suggest that based on the presentation of our reported case, ovarian derived carcinoid syndrome should be considered in the differential diagnosis of female patients presenting with new-onset, difficult to control hypertension.

Several studies have sought to determine significant risk factors for carcinoid tumors progressing to carcinoid heart disease. Bhattacharyya et al. found statistical significance for flushing three or more times per day and 5-HIAA of 300 or greater [12]. While Moller et al. found elevated urinary peak 5-HIAA and receiving chemotherapy increased the risk of carcinoid heart disease [13]. The relation of chemotherapy to valve disease is proposed to be due to therapy induced transient bursts of serotonin being released from the tumor [13]. The presence of carcinoid heart disease has been shown to increase in likelihood in cases where measured serotonin is higher and carcinoid syndrome symptoms are three times more likely in patient with cardiac involvement compared to those without valve disease [14]. In addition to higher serotonin levels causing worsening valvular disease, more significant pathology has been associated with higher levels of tachykinins, notably neuropeptide $\mathrm{K}$ and substance $\mathrm{P}$, which have been shown to stimulate fibroblast proliferation in vitro [15]. It has also been demonstrated that heart disease is related to the level of carcinoid tumor metabolite release as opposed to the duration of disease [15]. Thus, early diagnosis of carcinoid tumor may not lead to substantial prognostic improvement if the tumor is secreting particularly high levels of serotonin and related substances.

In addition to secretion of serotonin and tachykinins, the neuroendocrine morphological tumors have been known to produce a wide variety of neurohormones and endocrine products. These substances include neurotensin, ACTH, calcitonin, growth hormone and pancreatic enzymes [16-19]. Additionally, neuroendocrine tumors can present with malignant hypercalcemia, like other forms of solid and hematologic malignancies. Hypercalcemia occurs in approximately $20-30 \%$ of malignancies, most commonly through the secretion of parathyroid hormone-related protein (PTH-rP) [20]. Rarely, neuroendocrine tumors can induce hypercalcemia through production of 1,25 -dihydroxyvitamin $\mathrm{D}$, which is typically only associated with lymphomas [20]. These paraneoplastic syndromes have the potential to complicate the evaluation and diagnosis of carcinoid heart disease due to the increased possibility of multi-organ system patient presentations.

In one study, when the tumor is limited to a single ovary, surgical intervention resulted in excellent outcomes, however if the tumor has reached an advanced stage, the five-year survival rate falls to $33 \%$ [6]. In a large analysis of carcinoid tumors derived from all reported primary sites, the five-year survival rate was found to be $95 \%$ in localized cases and $13.3 \%$ in cases with metastases [4]. Higher pre-operative 5-HIAA has been associated with higher peri-operative mortality [21], indicating that pre-surgical evaluation should include tumor metabolites for risk stratification. It has been reported that somatostatin and hepatic artery embolization does not prevent progression of cardiac disease [13]. Patients who develop carcinoid heart disease may need valve replacement based on their clinical and echocardiographic features, however, no optimal timing of surgery related to severity of valve dysfunction or symptoms has been established [1]. Due to this lack of standardized management, these patients are managed on a case by case basis as determined by patient characteristics and clinician preferences.

No specific protocol has been established for post-operative surveillance of carcinoid syndrome patients with echocardiogram. Valve disease has been shown to progress even after tumor removal based on echocardiographic follow-up annually [11]. In a series of 80 patients, Mansencal et al. determined that all 80 patients developed heart disease by three years' post-operation of elevated 5-HIAA [11]. Due to the concern for progression of valvular disease during followup, we have continued to evaluate our case patient every year with echocardiogram, although her disease has not yet progressed.

\section{Conclusion}

The ovary is a highly unusual location for primary carcinoid tumors. Due to its exclusion from the portal venous circulation, ovarian carcinoid tumors can lead to carcinoid syndrome and carcinoid heart disease more rapidly and without hepatic metastases, which differentiates this condition from more typical gastrointestinal carcinoid tumors. In this case, we present a patient with primary ovarian neuroendocrine carcinoma with presenting symptoms most notable for hypertensive crises, which to the best of our knowledge has not been reported. Ovarian carcinoid tumors may be an under recognized cause of secondary hypertension in female patients. The comprehensive mechanism of carcinoid syndrome and carcinoid heart disease remains elusive, but high levels of serotonin, 5-HIAA, neuropeptide $\mathrm{K}$ and substance $\mathrm{P}$ have been implicated $[14,15]$. There are currently no established guidelines for treating carcinoid heart disease and monitoring patients for development or progression of valve disease. Further work in larger selections of patients may yield important conclusions regarding the most effective indications and timing of intervention as well as appropriate follow-up recommendations.

\section{Disclosures}

The authors report no sources of funding or conflicts of interest for this work. 


\section{Acknowledgements}

The authors have no acknowledgements to report for this work.

\section{References}

1. Bhattacharyya S, Davar J, Dreyfus G, Martyn E (2007) Caplin Carcinoid Heart Disease. Circulation 116: 2860-2865.

2. Bernheim AM, Connolly HM, Pellikka PA (2007) Carcinoid Heart Disease in Patients Without Hepatic Metastases. Am J Cardiol 99: 292-294.

3. Kulke MH, Mayer RJ (1999) Carcinoid tumors. N Engl J Med 340: 858-868. [Crossref]

4. Modlin IM, Sandor A (1997) An analysis of 8305 cases of carcinoid tumors. Cancer 79: 813-829. [Crossref]

5. Godwin JD 2nd (1975) Carcinoid tumors. An analysis of 2,837 cases. Cancer 36: 560569. [Crossref]

6. Davis KP, Hartmann LK, Keeney GL, Shapiro H (1996) Primary ovarian carcinoid tumors. Gynecol Oncol 61: 259-265. [Crossref]

7. Stewart M, Willis R, Saram G (1939) Argentaffin Carcinoma (Carcinoid Tumour) Arising in Ovarian Teratoma. Journal of Pathology and Bacteriology 49: 207-212.

8. Sauer WG, Dearing WH, Flock EV, Waugh JM, Dockerty MB, et al. (1958) Functioning carcinoid tumors. Gastroenterology 34: 216-230. [Crossref]

9. Chaowalit N, Connolly HM, Schaff HV, Webb MJ, Pellikka PA (2004) Carcinoid heart disease associated with primary ovarian carcinoid tumor. Am J Cardiol 93: 1314-1315. [Crossref]

10. Pellikka PA, Tajik AJ, Khandheria BK, Seward JB, Callahan JA, et al. (1993) Carcinoid heart disease. Clinical and echocardiographic spectrum in 74 patients. Circulation 87 : 1188-1196. [Crossref]

11. Mansencal N, Mitry E, Bachet JB, Rougier P, Dubourg O (2010) Echocardiographic follow-up of treated patients with carcinoid syndrome. Am J Cardiol 105: 1588-1591. [Crossref]
12. Bhattacharyya S (2001) Risk Factors for the Development and Progression of Carcinoid Heart Disease. Am J Cardiol 107: 1221-1226.

13. Møller JE, Connolly HM, Rubin J, Seward JB, Modesto K, et al. (2003) Factors associated with progression of carcinoid heart disease. N Engl J Med 348: 1005-1015. [Crossref]

14. Robiolo P (1995) Carcinoid Heart Disease: Correlation of High Serotonin Levels with Valvular Abnormalities Detected by Cardiac Catheterization and Echocardiography. Circulation 92: 790-795.

15. Lundin L (1998) Carcinoid Heart Disease: Relationship of Circulating Vasoactive Substances to Ultrasound-detectable Cardiac Abnormalities. Circulation 77: 264-269.

16. Yang K (1983) The Neuroendocrine Products of Intestinal Carcinoids. An Immunoperoxidase Study of 35 Carcinoid Tumors Stained for Serotonin and Eight Polypeptide Hormones. Cancer 51: 1918-1926.

17. Pearse AGE, Polak JM, Heath CM (1974) Polypeptide Hormone Production by "Carcinoid" Apudomas and Their Relevant Cytochemistry. Virchows Archive B Cell Pathology 16. [Crossref]

18. Klappenbach RS (1985) Composite Carcinoma- Carcinoid Tumors of the Gastrointestinal Tract: A Morphologic, Histochemical, and Immunocytochemical Study. Journal of Clinical Pathology 84: p. 137-143.

19. Lefebvre (1995) Subcutaneous Octreotide Treatment of a Growth Hormone-Releasing Hormone-Secreting Bronchial Carcinoid: Superiority of Continuous versus Intermittent Administration to Control Hormonal Secretion. European Journal of Endocrinology 133: 320-324. [Crossref]

20. Zhu V, de las Morenas A, Janicek M, Hartshorn K (2014) Hypercalcemia from Metastatic Pancreatic Neuroendocrine Tumor Secreting 1,25-Dihydroxyvitamin D. $J$ Gastrointest Oncol 5: 84-87. [Crossref]

21. Møller JE, Pellikka PA, Bernheim AM, Schaff HV, Rubin J, et al. (2005) Prognosis of carcinoid heart disease: analysis of 200 cases over two decades. Circulation 112: 3320-3327. [Crossref]

Copyright: (C2016 Brouillard AM. This is an open-access article distributed under the terms of the Creative Commons Attribution License, which permits unrestricted use, distribution, and reproduction in any medium, provided the original author and source are credited. 